\title{
Role of FGF-2 Transfected Bone Marrow Mesenchymal Stem Cells in Engineered Bone Tissue for Repair of Avascular Necrosis of Femoral Head in Rabbits
}

\author{
Fei Zhang Wu-xun Penga,b Lei Wang $^{a} \quad$ Jian Zhang ${ }^{a, b} \quad$ Wen-tao Dong ${ }^{a, b}$ \\ Jian-hua Wu ${ }^{a, b}$ Huai Zhang ${ }^{a, b}$ Jian-bo Wang ${ }^{a, b}$ Yin Zhao ${ }^{a, b}$ \\ aGuizhou Medical University, Guiyang, Guizhou, 'bepartment of Orthopedics, Affiliated Hospital of \\ Guizhou Medical University, Guiyang, Guizhou, China
}

\section{Key Words}

Avascular necrosis of the femoral head - Tissue engineering bone - Basic fibroblast growth factor $•$ Bone marrow mesenchymal stem cells

\begin{abstract}
Background/Aims: Avascular necrosis of the femoral head (ANFH) is the focus and difficulty of orthopedic diseases. Recently, tissue engineering bone for this disease has shown a good therapeutic effect. The aim of the present study was to investigate the therapeutic effect of basic fibroblast growth factor (FGF-2) as cytokines transfected bone marrow mesenchymal stem cells (BMSCs) in constructing tissue-engineered bone for avascular necrosis of the femoral head. Methods: The FGF-2 gene overexpressed lentivirus-transfected rBMSCs with xenogeneic antigen-extracted cancellous bone (XACB) to construct tissue engineered bone, and the model of early avascular necrosis of the femoral head was established by lipopolysaccharide (LPS) combined with hormone. The models were randomly divided into five groups: $A$ (control), B (XACB), C (XACB+rBMSCs), D (XACB+rBMSCs+Lv-GFP), and $E(X A C B+r B M S C s+L v-F G F-2 / G F P)$ groups. The therapeutic effect of the tissue engineered bone for the avascular necrosis of the femoral head was evaluated by gross anatomy, X-ray examination, immunohistochemistry and H\&E staining. Results: The FGF-2 gene was transfected into rBMSCs (Multiplicity of infection [MOI] $=100$ ) by lentivirus, and its efficiency was above $95 \%$. The rBMSCs were successfully transfected overexpressing FGF-2 by qPCR and western blot. After tissue engineering bone implantation, $\mathrm{X}$-ray examination and gross specimen observation revealed that the repair area in the $E$ group was $>80 \%$ at six weeks, the defect was completely repaired at 12 weeks, and osteogenesis was stronger, when compared with the other groups. For the X-ray score, vascular area density and new bone formation area were higher, when compared with the other groups, and the difference was statistically significant $(P<0.05)$. Conclusion: FGF-2 gene overexpression lentivirus transfection BMSCs combined with XACB to construct tissue engineered bone can effectively promote vascular regeneration, and improve the repair effect of avascular necrosis of the femoral head.
\end{abstract}




\section{Cellular Physiology Cell Physiol Biochem 2018:48:773-784 \begin{tabular}{l|l} 
and Biochemistry Published online: July 20, 2018 & $\begin{array}{l}\text { C) } 2018 \text { The Author(s). Published by S. Karger AG, Basel } \\
\text { www.karger.com/cpb }\end{array}$
\end{tabular} Zhang et al.: FGF-2/BMSCs/XACB for Repair ANFH}

\section{Introduction}

Avascular necrosis of the femoral head (ANFH) is a common disease of the hip joint, which is the first of all kinds of osteonecrosis, and this common in middle-aged people who are approximately 40 years old [1]. After the collapse of the femoral head, the acetabulum is involved, the pathological process cannot be reversed, and it is disabled by progressive destruction of the hip. Hence, pre-collapse treatment for ANFH has been the focus and difficulty of the Department of Orthopedics. Present treatment methods include surgical and non-surgical treatment. Non-operative treatment has a definite effect on osteonecrosis of the femoral head in the early stage [2], but more than $80 \%$ of ANFH cases still needs surgical treatment, including core decompression [3], osteotomy [4], and vascularized bone graft $[5,6]$. However, its pathogenesis remains unclear, and the treatment and curative effect of ANFH remains very limited.

In recent years, bone marrow mesenchymal stem cells (BMSCs) and basic fibroblast growth factor (bFGF) combined with xenogeneic antigen-extracted cancellous bone (XACB) were applied to repair early femoral head necrosis in bone tissue engineering in animal models, which achieved a good effect. However, the half-life of cytokines is short in vivo, requiring repeated administration [7]. The prsent study hypothesized and observed the novel therapeutic effect of the combined BMSCs-bFGF-XACB in bone tissue engineering to solve the burden of ANFH.

\section{Materials and Methods}

\section{Animals}

A total of 60 adult male and female New Zealand White rabbits (six months old, weighting 3.0-4.0 kg) and five young male New Zealand white rabbits (4-6 weeks old, weighting 1.0-2.0 kg) were provided by the Laboratory Animal Center of Guizhou Medical University (Guiyang, China). All procedures were performed in accordance with our Institutional Guidelines for Animal Research, and the investigation conformed to the Guide for the Care and Use of Laboratory Animals published by the US National Institutes of Health (NIH Publication No. 85-23, revised in 1996).

\section{Main materials and reagents}

Low glucose Dulbecco's modified Eagle's medium and fetal bovine serum (FBS; Gibco, USA), double antibody (Hyclon, USA); osteoporosis induction medium (Cyagen Biosciences Guangzhou Inc., China); ALP detection kit and puromycin (Beijing Solarbio Biotechnology Co., Ltd., China); bFGF gene overexpression lentivirus and lentivirus negative control (Shanghai Genechem Co., Ltd., China); Anti-CD44/FITC and Anti-bFGF antibody and Anti- $\beta$-acting antibody (Beijing Bioss Biotechnology Co., Ltd., China); Anti-CD34 antibody (Beijing Zsbio Co., Ltd., China).

\section{Isolation, culture and identification of rBMSCS}

The bone marrows of five young male New Zealand white rabbits (4-6 weeks old, weighting 1.0$2.0 \mathrm{~kg}$ ) were extracted under aseptic conditions. Cells were separated by density gradient centrifugation, and cultured in L-DMED medium (10\% FBS and $1 \%$ double antibody) at $37^{\circ} \mathrm{C}$ in a $5 \% \mathrm{CO}_{2}$ incubator. When primary cells converge reached $80 \%-90 \%$, they were passaged at $1: 3$. Cell growth was observed using an inverted phase contrast microscope (Guiyang Yong Chao Medical Electronic Technology Co., Ltd., China) every day. At the third generation good growth of BMSCs, surface antigen CD44 was identified by flow cytometry. Then, the osteogenic differentiation of BMSCs was induced by induction medium, and the osteogenic differentiation potential was identified by alkaline phosphatase staining and alizarin red staining.

Biocompatibility of XACB and rBMSCs

Third generation rBMSCs were inoculated in XACB for composite culture, and a piece of tissue engineered bone was removed every day. The growth curve was detected using CCK-8 method, and the 


\section{Cellular Physiology Cell Physiol Biochem 2018;48:773-784 \\ \begin{tabular}{l|l} 
and Biochemistry Published online: July 20, 2018 & $\begin{array}{l}\text { D } 2018 \text { The Author(s). Published by S. Karger AG, Basel } \\
\text { www.karger.com/cpb }\end{array}$ \\
\hline
\end{tabular} \\ Zhang et al.: FGF-2/BMSCS/XACB for Repair ANFH}

optimal time of tissue engineered bone implantation was determined. The tissue engineered bone were taken at sixth days after composite culture, cell surface attachment occurred, and the distribution and growth were observed using a scanning electron microscope.

\section{Lv-FGF-2 gene transfection and expression of FGF-2 in transfected rBMSCS}

The good growth of third generation rBMSCs were divided into three groups according to the preexperiment: A (control), B (rBMSCs+Lv-GFP), and C (rMSCs+Lv-FGF2/GFP). This was performed to find the best MOI(100) and the optimal transfection condition (Eni.s. and Polybrane). Then, lentivirus transfection was performed. After 10 hours, cells were observed and fresh medium was replaced. Three days after the transfection, the expression of the fluorescent protein was observed using an inverted fluorescence microscope, and transfection efficiency was calculated. At five days after the transfection, the puromycin medium was screened to obtain stable cell lines. The expression of the FGF-2 gene was detected by qPCR and western blot.

\section{Establishment of the ANFH model}

A total of 60 adult male and female New Zealand White rabbits (six months-old, weighting 3.0-4.0 kg) were routinely weighed and disinfected, and $10 \mathrm{ug} / \mathrm{kg} / \mathrm{d}$ of endotoxin was intravenously injected for two days, On the second day after the endotoxin injection, $40 \mathrm{mg} / \mathrm{kg} / \mathrm{d}$ of methylprednisolone was injected into the gluteal muscle for three days to establish the model of ANFH [8]. The imaging examination was able to prove that the model was successful. Then, the tissue engineering bone repair experiment was used.

The successful models of $45 \mathrm{New}$ Zealand rabbits were randomly divided into five groups, with nine rabbits in each group: A (control), B (XACB), C (XACB+rBMSCs), D (XACB+rBMSCs+Lv-GFP), and E (XACB+rBMSCs+Lv-FGF2/GFP). After using $1 \mathrm{mg} / \mathrm{Kg}$ of 3\% sodium pentobarbital for ear vein anesthesia, the hip was exposed under aseptic conditions and drilled at the junction of the bone and cartilage. The necrotic bone tissue in the femoral head was scraped with a curette. In the experimental group, the engineered bone tissue was transplanted on the right side, while the left side was self contrast. At preoperative 30 minutes and three days after surgery, cefuroxime sodium ( $80 \mathrm{mg} / \mathrm{Kg} / \mathrm{d}$ ) gluteal injection was given to prevent infection.

\section{Evaluation of repair effect}

Animal observation, X-ray examination and scoring

Animal weight, diet, defecation, activity and wound healing after surgery were determine to observe the behavioral state. At postoperative 3, 6 and 12 weeks, X-ray examination was respectively performed to evaluate the repair effect. The evaluation criteria were divided into four levels: excellent, good, fair, and poor. The corresponding scores were 4, 3, 2 and 1 [9].

\section{Observation of femoral head specimens}

At 3, 6 and 12 weeks after the operation, three rabbits were sacrificed in each group. The femoral head was removed under aseptic condition. The surface of the femoral head was observed, and dissected longitudinally from the middle of the femoral head, and the repair of femoral head defect was observed.

\section{Immunohistochemical staining and vascular area density analysis}

Immunohistochemical staining was performed on specimens with the CD34 polyclonal antibody. A slice was randomly selected for each sample at each time point, three 100 -fold fields of vision were randomly selected for each slice, and the blood vessels were counted for each field of vision and average for each sample. Then, the HPIAS-1000 high definition color pathological picture report analysis system was used to detect the density of the vascular area. Vascular area density was equal to the area of the vessel divided by the area of the statistics, and multiplied by $100 \%$. That is, vascular area density = the vessel area / the entire visual field $\times 100 \%$.

\section{H\&E staining and new bone formation area}

After the specimens were decalcified, these were prepared into 3- $\mu$ m thick slices, stained with H\&E and observed using a microscope. A slice was randomly selected for each sample at each time point. In each bone graft area (or the defect area of the control group), three 100 -folds visual fields were randomly 


\section{Cellular Physiology Cell Physiol Biochem 2018;48:773-784 \begin{tabular}{ll|l} 
and Biochemistry Published online: July 20, 2018 & $\begin{array}{l}\text { (c) } 2018 \text { The Author(s). Published by S. Karger AG, Basel } \\
\text { www.karger.com/cpb }\end{array}$
\end{tabular} \\ Zhang et al.: FGF-2/BMSCS/XACB for Repair ANFH}

selected to observe the area of new bone formation. The HPIAS-1000 high definition color pathological picture report analysis system was used to analyze the image and calculate the area percentage of the new bone formation, and the percentage of the new bone formation area = new bone area $/$ the entire visual field.

\section{Results}

\section{Extraction and identification of rBMSCS}

The primary rBMSCs adhered and stretched after 24 hours, and the shape was irregular (Fig. 1A). After approximately five days, the cells became colonized (Fig. 1B). After approximately 10 days, primary cells were gradually covered approximately $80 \%-90 \%$ of the bottom (Fig. 1C). After the passage of cells, the shape tended to be single, cells were mainly long spindle, proliferation was more active, and cells could pass a generation at approximately three days (Fig. 1D). Third generation rBMSCs were identified by flow cytometry, and CD44 positive cells were $>99 \%$ (Fig. $2)$. The rBMSCs were cultured in osteogenic induction medium, and calcium nodules were formed in 14 days. Alizarin red staining and alkaline phosphatase staining were positive (Fig. 3).

\section{Biocompatibility of XACB and rBMSCS}

The rBMSCs were incubated with XACB to detect their biocompatibility. There was no significant change in cell morphology when observed under an inverted microscope, showing a long spindle-shape and few polygonal shapes (Fig. 4A). For the composite culture at the sixth day, scanning electron microscopy revealed cells with extending pseudopods that attached to XACB (Fig. 4B). The growth curve was plotted using the CCK- 8 method. The results revealed that cells did not significantly grow on the first day of the compound culture, but began to grow exponentially on the second day. However, on the sixth day, it gradually reached a plateau (Fig. 4C).

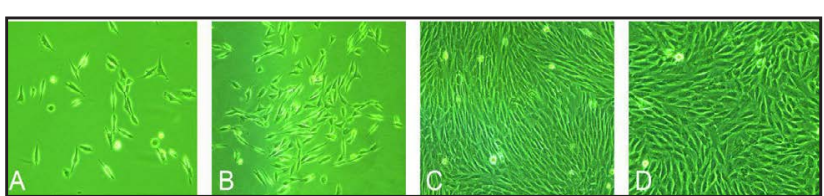

Fig. 1. Growth of rBMSCs observed using an inverted phase contrast microscope Note: (A) primary rBMSCs was cultured for 24 hours $(\times 100)$; (B) primary rBMSCs were cultured for five days $(\times 100)$; $(C)$ primary rBMSCs was cultured for 10 days $(\times 100)$; (D) third generation rBMSCs $(\times 100)$.

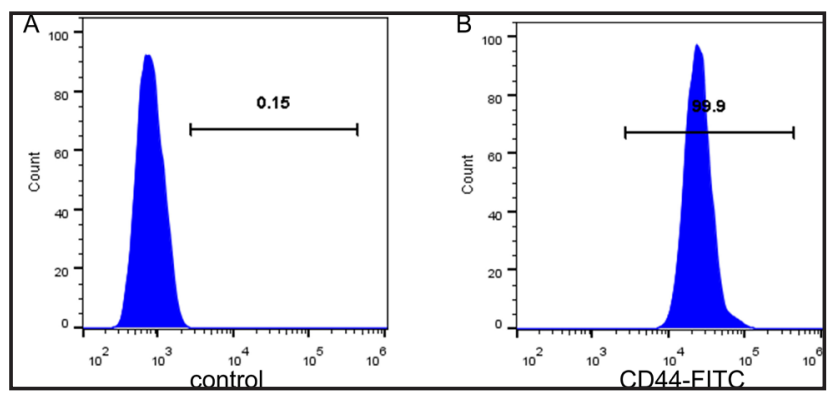

Fig. 2. Identification of rBMSC surface antigen by flow cytometry Note: (A) the positive rate of CD44 in the control group was approximately $0.15 \%$; (B) the positive rate of CD44 in the experimental group was $>99 \%$.

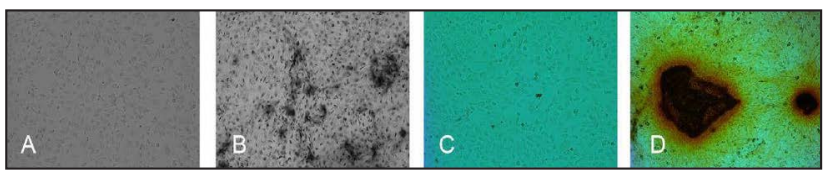

Fig. 3. After two weeks of osteogenic induction of BMSCs, alizarin red and alkaline phosphatase staining. Alkaline phosphatase staining: (A) control group, alkaline phosphatase staining was negative $(\times 100)$; $(B)$ test group, alkaline phosphatase staining, most of the cytoplasm of cells was found with the deposition of black particles $(\times 100)$. Alizarin red staining: $(C)$ control group, no calcium nodule formation, alizarin red staining negative $(\times 100)$; (D) experimental group, cells had stratified growth, calcium nodules were visible $(\times 100)$. 


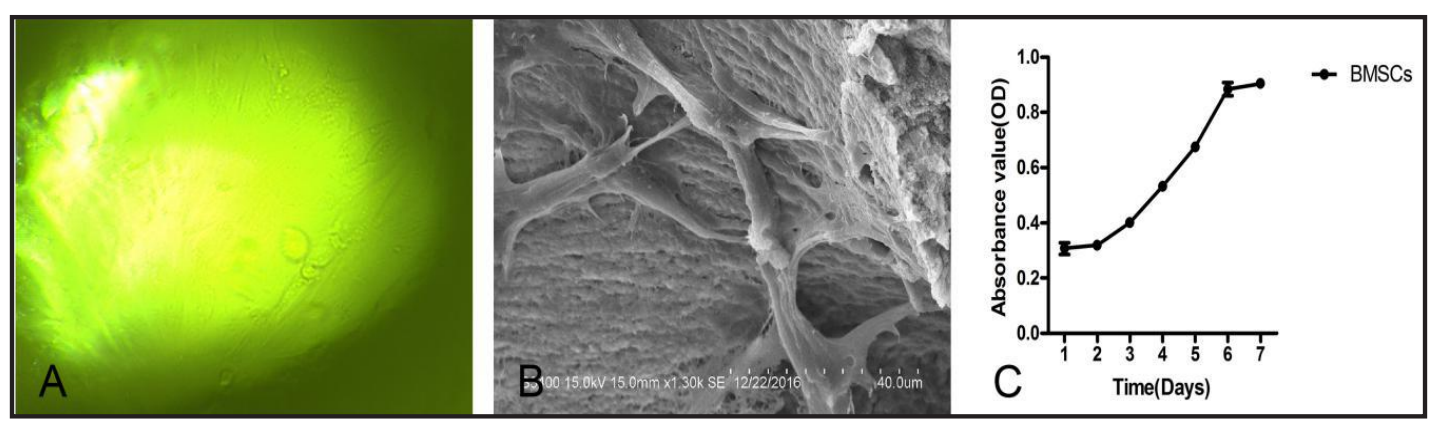

Fig. 4. Biocompatibility detection of rBMSCs and XACB: (A) composite cultured for six days, and cell growth observed under an inverted microscope $(\times 100)$; (B) composite culture for sixth days, cells grew and attached to XACB when observed by scanning electron microscopy; (C) the CCK-8 method was used to detect the growth curve of rBMSCs and the XACB composite culture.

FGF-2 gene transfectedBMSCs and its detection

The expression of fluorescent protein was observed using an inverted fluorescence microscope, and the best MOI was determined. Third generation BMSCs were transfected with the FGF-2 gene overexpression lentivirus. At three days after transfection, the fluorescent protein was detected under an inverted fluorescent microscope. It was found that it reached its peak at approximately the fifth day. MOI=100, and the highest transfection efficiency was above 95\%, which was significantly higher than MOI $=10$ or $50 \quad(P<0.001)$, compared with MOI $=200$ or 300 , but there was no significant difference $(P>0.05)$. However, when MOI=200 or 300 , the cell body increased and growth became slow. These phenomena indicate that the lentivirus was more toxic to cells after transfection. Hence, 100 was chosen as the best MOI value (Fig. 5).

The mRNA expression of FGF-2 was detected by qPCR. After the transfection of the FGF-2 gene in BMSCs, stable strains were screened,
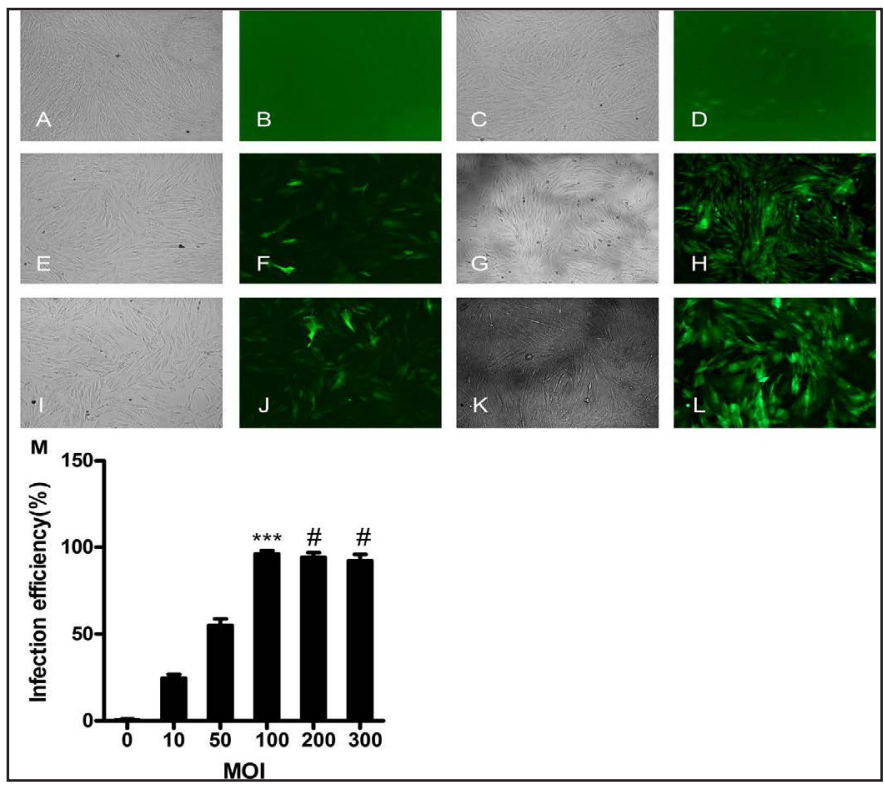

Fig. 5. The expression of fluorescent protein and the transfection efficiency of different MOI were observed by inverted fluorescence microscopy on the $5^{\text {th }}$ day after transfection. Note: MOI = 0 (without virus), cells grew well in the bright field, no fluorescence protein expression ( $A$ and $\mathrm{B}$ ); $\mathrm{MOI}=10$, shows that a small amount of cells had fluorescent protein expression ( $\mathrm{C}$ and D); MOI=50, the majority of cells weakly expressed fluorescent protein $(E$ and $F) ; \mathrm{MOI}=100$, shows that cells grew well, no significant changes in morphology, a large number of cells strongly expressed fluorescent protein, transfection efficiency was $>95 \%$ ( $\mathrm{G}$ and $\mathrm{H}$ ); MOI $=200$ or 300 , most cells were strongly expressed with fluorescent protein, transfection efficiency was $>90 \%$, but the cell volume and dead cells increased (I, J, K and L); (M) Comparison of transfection efficiencies with different MOI values. ${ }^{* * *} \mathrm{P}<0.001$ vs. the $\mathrm{MOI}=0,10$, or 50 group, $\mathrm{n}=3$ per group; \# $\mathrm{P}>0.05$ vs. the $\mathrm{MOI}=200$ or 300 group, $\mathrm{n}=3$ per group. 
and the expression of FGF-2 mRNA was detected by qPCR. The amplification curve revealed the logarithmic growth stage and plateau stage (Fig. 6A). These results show that the FGF-2 gene was expressed in each group, and the expression in the $\mathrm{C}$ group was significantly higher than that in the $A$ and $B$ groups $(P<0.001$, Fig. 6B).

Western blot was used to detect the protein expression of FGF-2. With positive bands at approximately $17 \mathrm{Kd}$, the FGF-2 gene was expressed in each group (Fig. 6C). The expression in the $\mathrm{C}$ group was significantly higher than that in the A or B group $(P<0.05$, Fig. 6D).

Tissue engineering bone transplantation

The model of ANFH was successfully prepared by LPS and methylprednisolone, and the tissue engineered bone was successfully implanted after core decompression (Fig. 7).

\section{Observation of live animals}

All the experimental animals started eating on the second day after the operation, the behavior was good. One case of blank superficial infection was found at one week after operation, and the incision was healed after debridement and antibiotic treatment (B group). The remaining experimental animals were underwent wound healing, and no any animal death was recorded during the observation period.

\section{Tissue specimen observations}

At three weeks after surgery: In the A group, the defect area and surrounding bone tissue boundaries were clear, and this was filled with granulation tissue without obvious new bone formation (Fig. 8A). In the B, C and D groups, XACB absorption in the defect area was not complete, the defect area was clear, and a small amount of new bone was formed around it (Figs. 8B, 8C and 8D). In the E group, XACB was not completely absorbed, and new bone formed (Fig. 8E).

At six weeks after the operation: In the A group, the defect area and the surrounding bone tissue boundary remained clear, and no obvious new bone formation occurred (Fig. $8 \mathrm{~F}$ ). In the B group, XACB absorption was not complete, the defect area boundary was clear, and a small amount of new bone formation was observed (Fig. 8G). In the C and D groups, the defect area remained visible and new bone formed at the margin (Figs. 8H and 8I). In group E, most of the defect areas were filled with new bone, the boundary of the defect area was blurred, few granulation tissues were found in the central part, and the XACB was almost completely absorbed (Fig. 8J). 
At 12 weeks after surgery: In the $\mathrm{A}$ group, the defect area was almost filled with granulation tissue, and a small amount of new bone formation could be observed (Fig. 8K). In the $\mathrm{B}$ group, the defect area and the surrounding normal bone boundary was clear, and visible new bone formation could be observed (Fig. 8L). In the $\mathrm{C}$ and $\mathrm{D}$ groups, the defective area XACB was completely absorbed, and this was surrounded by a large number of new bone formations (Figs. 8M and 8N). In the $\mathrm{E}$ group, the defective area was completely repaired (Fig. 80).

\section{X-ray observation and scoring}

At three weeks after operation: There was a sclerosis zone around the defect area, and no new bone formation was found in the A group (Fig. $9 A)$. In the $B, C$ and $D$ groups, the low density bone formation around the defect area was approximately $10-30 \%$ of the
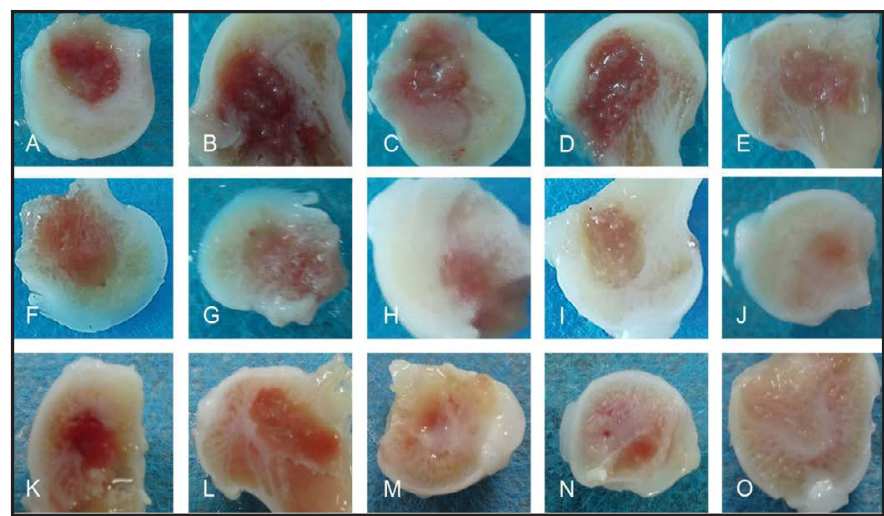

Fig. 8. Observation of gross specimens at 3, 6 and 12 weeks after the operation: At three weeks after the operation, the defects in the $\mathrm{A}, \mathrm{B}, \mathrm{C}$ and $\mathrm{D}$ groups were filled with granulation tissue. The formation of new bone was not obvious (Fig.s 8A, 8B, 8C and 8D), and new bone formation was observed in E group (Fig. 8E). At six weeks after the operation, the defect area in the A group was filled with granulation tissue without obvious new bone formation (Fig. 8F). The new bone formation was found in the defect areas in the $\mathrm{B}, \mathrm{C}$ and $\mathrm{D}$ groups (Fig.s $8 \mathrm{G}, 8 \mathrm{H}$ and $8 \mathrm{I}$ ), but the osteogenesis was weaker than that in the E group (Fig. 8J). At 12 weeks after the operation, there were a few new bone formations in the defect area in the A group (Fig. 8K). A large number of new bone formations were found in the defect areas in the $B, C$ and D groups, but these were not completely repaired (Fig.s 8L, 8M and $8 \mathrm{~N}$ ). The defect area in the E group was completely repaired (Fig. 80).
Fig. 9. X-ray observation and X ray score at 3, 6 and 12 weeks after the operation: At three weeks after the operation, there was no obvious osteogenesis in each group, and there was no significant difference in X-ray score (Fig.s 9A-9E and 9P). At postoperative six and 12 weeks, the osteogenesis in the A group was the weakest, and the X-ray score was the lowest, " $\mathrm{P}<0.05$ vs. the A group, $\mathrm{n}=3$ 5 per group. In the $\mathrm{E}$ group, bone formation were stronger than the other groups, and had the highest score of $\mathrm{X}$-ray, ${ }^{*} \mathrm{P}<0.05$ vs. the $\mathrm{A}, \mathrm{B}, \mathrm{C}$ and $D$ groups, $n=3-5$ per group (Fig.s 9F-9P).
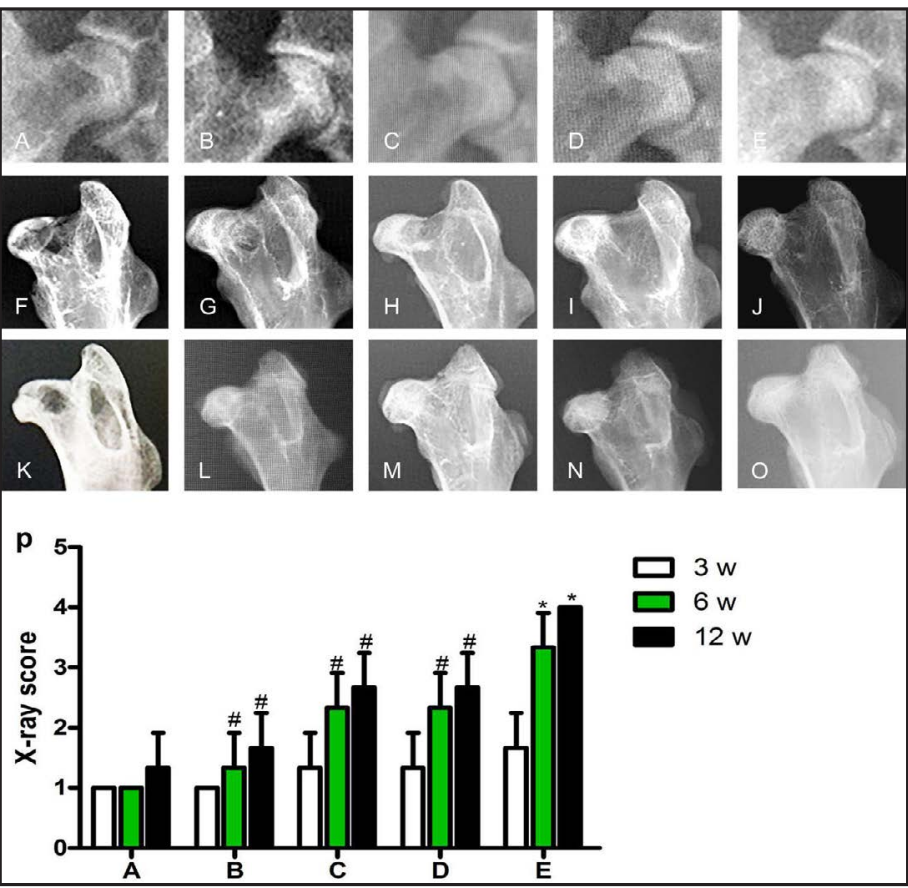
defect (Figs. 9B, 9C and 9D). A small amount of new bone formation was observed around the defective area in the E group, and approximately $20-40 \%$ new bone filling defects was observed (Fig. 9E).

At six weeks after the operation: In the A group, the boundaries were clear and no new bone formation was observed (Fig. 9F). In group B, the defect area was still clear, showing a small amount of new bone formation. Furthermore, new bone filling defects were approximately 20-30\%, and almost discontinuous low-density bones were observed (Fig. 9G). In the $C$ and $D$ groups, new bone filling defects were approximately 50-80\% (Fig. $9 \mathrm{H}$ and $9 \mathrm{I}$ ). In the E group, most of the defect area were filled with new bone (approximately $80-97 \%$ ), and most of which were normal density bones, and the surrounding boundaries were blurred (Fig. 9J).

At postoperative 12 weeks: The boundary between the defective area and the surrounding bone tissue in the A group was still clear, and low density bone was found in the defect (Fig. 9K). The defect of the new bone was approximately $25-40 \%$ in the B group, and most were discontinuous low density bones (Fig. 9L). In the $\mathrm{C}$ and $\mathrm{D}$ group, the new bone filling defect of approximately 60-90\%, and most were normal density bones (Figures $9 \mathrm{M}$ and $9 \mathrm{~N}$ ). In the E group, defective area was completely repaired (Fig. 90). At six and 12 weeks after the operation, the X-ray score in the E group was significantly higher than that in the other groups $(P<0.05)$. Furthermore, this was higher in the $\mathrm{C}$ and $\mathrm{D}$ groups, compared with that in the $A$ and $B$ groups $(P<0.05)$, but there was no statistical significance between the $\mathrm{B}$ and $\mathrm{A}$ group, and the $\mathrm{D}$ and $\mathrm{C}$ groups $(P>0.05$, Fig. $9 \mathrm{P})$.

\section{CD34 immunohistochemical staining and angiogenesis area}

At three weeks after the operation: The staining of each group was positive, and the capillary density of each group increased with the prolongation of time. However, the A and $B$ groups had the least at each time point, and the area of the blood vessel was lower than that of the other groups $(P<0.05)$. At postoperative six and 12 weeks: The capillary density of the C, D and E groups were significantly increased, and the anastomosis was net. The density of blood vessels in the E group was the highest, which was higher than that of the other groups $(P<0.05)$, and there was no significant difference between the $\mathrm{C}$ and $\mathrm{D}$ groups $(P>0.05$, Fig. 10).

\section{H\&E staining and analysis of the new bone formation area}

At three weeks after the operation: There were a large number of fibrous connective tissue in the defect area in the A group, showing a small amount of capillaries, with no osteoid tissue formation (Fig. 11A). In the B, C and D groups, fibrous connective tissue growth occurred along the XACB, which revealed the capillaries, and the XACB surface could be observed with a small amount of bone-like tissue formation (Figs. 11B, 11C and 11D). In the E group, there were more capillaries in fibrous connective tissues, more fibrous callus formed, and the new bone formation area was higher than the other groups $(P<0.05$, Figs. 11E and 11P).

At six weeks after the operation: There were no new bone formations in the defective area in the A (Fig. 11F). The XACB in the B group was not completely absorbed, and the osteoid tissue was formed on the surface with visible capillaries (Fig. 11G). In the C and D groups, a large number of osteoid tissues were found in the defect area, most of these were immature bone, the number of capillaries increased, and the area of new bone formation was higher than that in the A and B groups $(P<0.05$; Figs. $11 \mathrm{H}, 11 \mathrm{I}$ and $11 \mathrm{P})$. Most of the $\mathrm{XACB}$ in the E group were replaced by newborn bones, most of these were mature bone tissues, and a large number of osteoblasts and osteoclasts were found on the surface. The area of the new bone was higher than that of the other groups $(P<0.05$; Figs. 11J and 11P).

At postoperative 12 weeks: A small number of bone tissues were observed in the defect area in the A group, and the capillary was scattered in the fibrous tissue (Fig. 11K). The number of bone-like tissues in the defective area in the B group was larger than that before, and the capillaries were visible in the fibrous tissue (Fig. 11L). In the $\mathrm{C}$ and $\mathrm{D}$ groups, a large 


\begin{tabular}{|c|c|c|}
\hline \multirow{2}{*}{$\begin{array}{c}\text { Cellular Physiology } \\
\text { and Biochemistry }\end{array}$} & \multicolumn{2}{|c|}{ Cell Physiol Biochem 2018;48:773-784 } \\
\hline & $\begin{array}{l}\text { DOI: 10.1159/000491906 } \\
\text { Published online: July 20, } 2018\end{array}$ & $\begin{array}{l}\text { O } 2018 \text { The Author(s). Published by S. Karger AG, Basel } \\
\text { www.karger.com/cpp }\end{array}$ \\
\hline
\end{tabular}

Fig. 10. CD34 immunohistochemical staining and capillary area density at 3, 6 and 12 weeks after the operation: At all time points, CD34 staining was positive. At 6 and 12 weeks after the operation, the capillary anastomosis in the C, D and E groups was net (Fig.s 10A-100). At each time point, the capillary area density was higher in the $\mathrm{E}$ group than in the other groups, ${ }^{*} \mathrm{P}<0.05$ vs. the $\mathrm{A}, \mathrm{B}, \mathrm{C}$ and $\mathrm{D}$ groups, $n=3-5$ per group. There was no significant difference between the $A$ and $B$ groups, and $C$ and $D$ groups, ${ }^{\#} \mathrm{P}>0.05, \mathrm{n}=3-5$ per group. However, this was higher in the $\mathrm{C}$ and $\mathrm{D}$ groups, than in the $A$ and $B$ groups, ${ }^{*} \mathrm{P}<0.05$ vs. the $A$ and $B$ groups, $n=3-5$ per group (Fig. 10P).

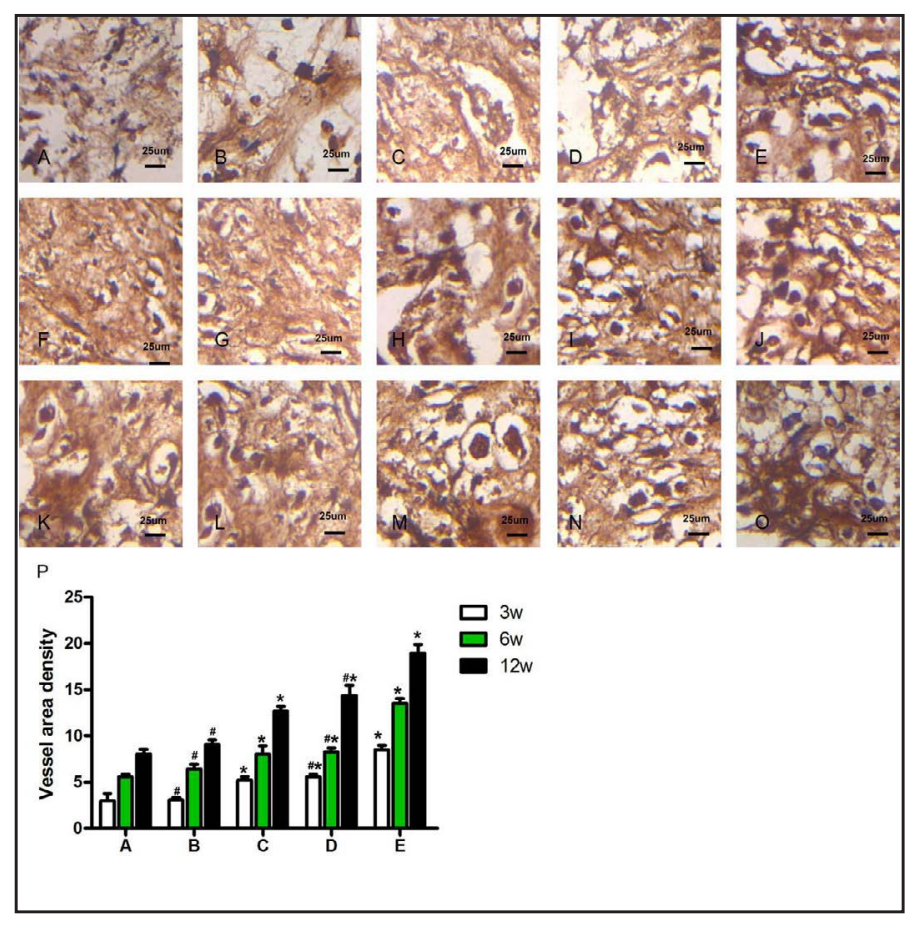

Fig. 11. H\&E staining and new bone formation area at 3, 6 and 12 weeks after the operation: At three weeks after the operation, all defects were dominated by fibrous connective tissue, and osteoid tissues formed in the E group (Fig.s 11A-11E). At six weeks after the operation, bone like tissue formed in the $\mathrm{B}, \mathrm{C}$ and $\mathrm{D}$ groups. In the $\mathrm{E}$ group, bone tissue in the defect area increased, and more capillaries were observed (Fig.s 11F-11J). At 12 weeks after the operation, XACB in the E group was completely absorbed, the mature bone tissues and bone marrow tissues were observed, while the other groups were immature (Fig.s $11 \mathrm{~K}-110)$. After 3, 6 and 12 weeks,

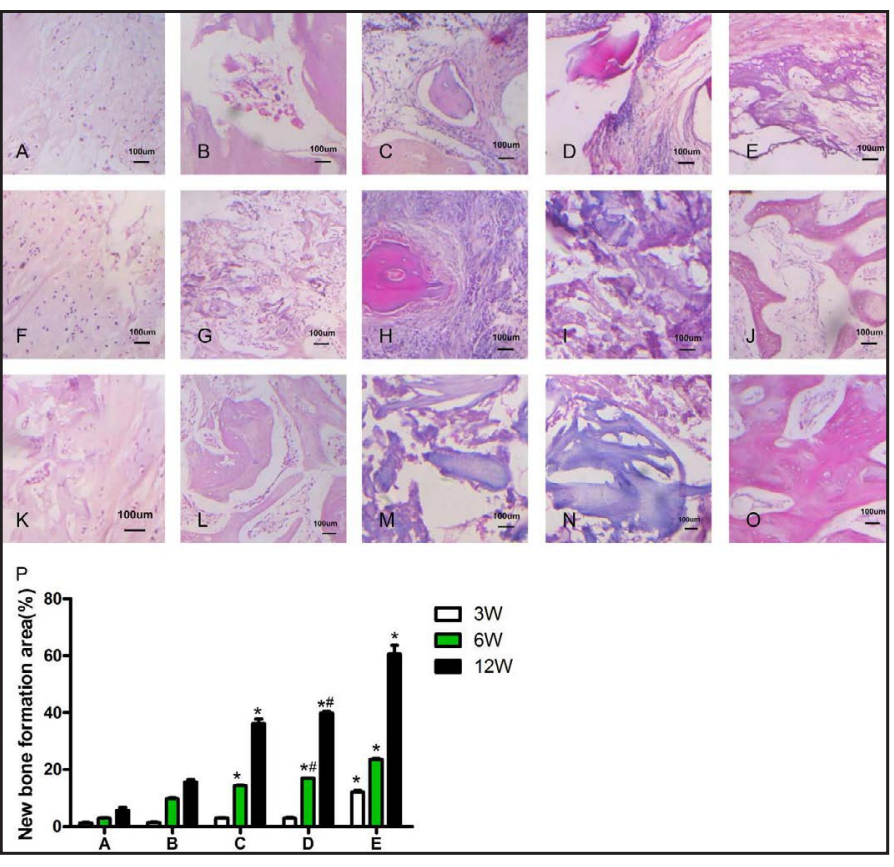
the new bone formation area of the $\mathrm{E}$ group was higher than that of the other groups, ${ }^{*} \mathrm{P}<0.05$ vs. the $\mathrm{A}, \mathrm{B}, \mathrm{C}$ and $\mathrm{D}$ groups, $\mathrm{n}=3-5$ per group. After six and 12 weeks, the new bone formation area in the $C$ and $D$ groups was not statistically significant, ${ }^{\text {}} \mathrm{P}>0.05, \mathrm{n}=3-5$ per group, but was higher than that in the A and B groups, ${ }^{*} \mathrm{P}<0.05$ vs. the $\mathrm{A}$ and $\mathrm{B}$ groups, $\mathrm{n}=3-5$ per group (Fig. 11P).

number of new bones in the defect area were formed by creeping substitution, osteoblasts and osteoclasts were found, and the number of new blood vessels increased. The new

\section{KARGER}


bone formation area was higher than that in the A and B group $(P<0.05)$, and there was no statistical significance between the $\mathrm{D}$ and $\mathrm{C}$ groups $(P>0.05$; Figs. $11 \mathrm{M}, 11 \mathrm{~N}$ and $11 \mathrm{P})$. In the E group, the XACB in the defect area was completely absorbed, and the mature bone tissue and bone marrow tissue were found, and the new bone formation area was the highest $(P<0.05$; Figs. 110 and 11P).

\section{Discussion}

The present study used the gene transfection technique combined with tissue engineering technology, and provided strong evidence for the cure of early ANFH. Endotoxin combined with hormone was used to successfully establish the rabbit model of ANFH, and the FGF-2 gene was transfected into rBMSCs by lentivirus and cultured with XACB to construct the tissue-engineered bone graft for the repair of the rabbit model. Through gross anatomy observation, X-ray examination, H\&E staining and immunohistochemistry, it was confirmed that the defect in the FGF-2 gene transfection group was completely repaired at 12 weeks after the operation, compared with the other groups. This obviously promoted angiogenesis and osteogenesis in the ANFH, and significantly improved the efficacy of ANFH.

The main pathological change of ANFH was the death of bone cells caused by ischemia $[10,11]$. Therefore, vascular regeneration and bone remodeling are critical for ANFH [12]. In recent years, tissue engineering technology has been used in the treatment of ANFH, which can promote vascular regeneration and bone reconstruction. Similarly, in the present study, it was found that the repair effect in the decompression group was significantly lower than the other experimental groups. Furthermore, these results also revealed that the tissue engineered bone could effectively promote the repair of ANFH, and this is consistent with most literature reports [13-16].

In treatment, the tissue engineered bone must include the basic elements of bone defect repair: seed cells, scaffold materials and cytokines. XACB and BMSCs are commonly used as cell scaffolds and seed cells, and have been widely used. However, the key to regulate seed cells is the cytokine. Some studies have reported that BMP-2 can be used as a cytokine to construct tissue engineered bone, which can promote the osteogenesis of bone marrow stromal cells to improve the repair of ANFH. However, this had no obvious effect on the regeneration of blood vessels in ischemic necrosis [17], and has the risk of heterotopic ossification [18]. In 2016, Zhang Hao-Xuan et al. [19] reported the use of VEGF combined with BMP-2 double factor to construct tissue engineered bone for ANFH while promoting osteogenesis. This also promoted the revascularization, which significantly improved the therapeutic effect of ANFH. However, in 2016, Li Bin et al. [20] reported that the FGF-2 single factor construction of tissue engineered bone repaired the ANFH, and also promoted osteogenesis and angiogenesis. Furthermore, FGF- 2 can promote the proliferation of BMSCs [21]. FGF-2 was used as a cytokine through the gene transfection BMSCs to construct a tissue engineered bone graft for the repair of the rabbit femoral head necrosis model, and the results revealed that in the FGF-2 gene transfection group, the defect repair more than approximately $80 \%$ at six weeks after the operation, and the defect was fully restored at 12 weeks. At each time point, the density of the vascular area and the area of the new bone formation in the necrotic area were significantly higher than those in the other groups, which also confirms that FGF-2 has a dual role in promoting angiogenesis and osteogenesis.

FGF-2, as a cytokine to construct tissue engineered bone, can effectively promote the repair of ANFH, but it still has shortcomings, such as the half-life is short in vivo, repeated administration, and the high-dose administration has adverse reactions [22]. For this problem, FGF-2 gene overexpression lentivirus was used to transfect BMSCs, and the sustained overexpression of FGF-2 protein was observed. These results revealed that the FGF-2 gene could be effectively integrated into the cell genome after the transfection 


\section{Cellular Physiology Cell Physiol Biochem 2018;48:773-784 \begin{tabular}{l|l} 
DOI: 10.1159/000491906 & O 2018 The Author(s). Published by S. Karger AG, Basel \\
www.karger.com/cpb
\end{tabular} \\ Zhang et al.: FGF-2/BMSCs/XACB for Repair ANFH}

of BMSCs by the lentivirus, thereby achieving the sustained and stable overexpression. Therefore, the use of gene transfection can effectively solve this shortcoming.

However, it should be noted that BMSCs can be expressed the FGF-2 protein itself. The limitation of the present study is that the FGF-2 gene of BMSCs were not treated through the suppression of the expression of related technologies, such as RNAi technology. Thus, the reverse to prove that FGF-2 has to promote BMSC proliferation, vascular regeneration and osteogenesis would make this experiment more stringent.

In conclusion, tissue engineering technology combined with gene transfection technology, and the use of FGF-2 gene overexpression lentivirus to transfect BMSCs, combined with XACB to construct tissue engineered bone grafts and repair ANFH, can not only solves the shortcomings, in which FGF-2 has a short half-life in vivo and need repeated administration, but also effectively promotes angiogenesis and osteogenesis in the ischemic necrosis area, significantly improving the repair effect of ANFH.

\section{Acknowledgements}

This work were supported by grant from the Guizhou Provincial Health and Family Planning Commission Fund Projects (No. gzwjkj2016-1-001, gzwjkj2018-1-008), Guizhou Provincial Science and Technology Fund Project (No. [2017]7197), Guiyang Science and Technology Fund Project (No. [2017]5-2). FZ:designed of the study, acquisition of the data, analysis and interpretation of data, drafting of the article and final approval of the article. WXP and LW: design of the study, analysis and interpretation of data, critical revision of the study for important intellectual content, and final approval of the manuscript. JZ, WTD and JHW: interpretation of data, critical revision of article for important intellectual content, and final approval of manuscript. HZ, JBW and YZ: acquisition of the data, analysis and interpretation of the data, and final approval of the article.

\section{Disclosure Statement}

The authors declare that there are no conflicts of interest.

\section{References}

1 Wen Q, Zhou C, Luo W, Zhou M, Ma L: Pro-osteogenic effects of fibrin glue in treatment of avascular necrosis of the femoral head in vivo by hepatocyte growth factor-transgenic mesenchymal stem cells. J Transl Med 2014;12:114.

-2 Han Y, Lee JK, Lee BY, Kee HS, Jung KI, Yoon SR: Correction: Effectiveness of Lower Energy Density Extracorporeal Shock Wave Therapy in the Early Stage of Avascular Necrosis of the Femoral Head. Ann Rehabil Med 2017;41:337-338.

- Calori GM, Mazza E, Colombo A, Mazzola S, Colombo M: Core decompression and biotechnologies in the treatment of avascular necrosis of the femoral head. EFORT Open Rev 2017;2:41-50.

-4 Takegami Y, Komatsu D, Seki T, Ishiguro N, Hasegawa Y: Total hip arthroplasty after failed curved intertrochanteric varus osteotomy for avascular necrosis of the femoral head. Nagoya J Med Sci 2016;78:89-97.

5 Gagala J, Tarczyńska M, Gawęda K: Clinical and radiological outcomes of treatment of avascular necrosis of the femoral head using autologous osteochondral transfer (mosaicplasty): preliminary report. Int Orthop 2013;37:1239-1244.

6 Gao YS, Chen SB, Jin DX, Sheng JG, Cheng XG, Zhang CQ: Modified surgical techniques of free vascularized fibular grafting for treatment of the osteonecrosis of femoral head: Results from a series of 407 cases. Microsurgery 2013;33:646-651. 


\section{Cellular Physiology Cell Physiol Biochem 2018;48:773-784 \begin{tabular}{l|l|l} 
and Biochemistry $10.1159 / 000491906$ & $\begin{array}{l}\text { D } 2018 \text { The Author(s). Published by S. Karger AG, Basel } \\
\text { www.karger.com/cpb }\end{array}$
\end{tabular} \\ Zhang et al.: FGF-2/BMSCs/XACB for Repair ANFH}

7 Chen B, Li B, Qi YJ, Ni QB, Pan ZQ, Wang H, Chen LB: Enhancement of tendon-to-bone healing after anterior cruciate ligament reconstruction using bone marrow-derived mesenchymal stem cells genetically modified with bFGF/BMP2. Sci Rep 2016;6:25940.

8 Wang T, Teng S, Zhang Y, Wang F, Ding H, Guo L: Role of mesenchymal stem cells on differentiation in steroid-induced avascular necrosis of the femoral head. Exp Ther Med 2017;13:669-675.

-9 Yamada Y, Nakamura S, Ito K, Sugito T, Yoshimi R, Nagasaka T, Ueda M: A feasibility of useful cell-based therapy by bone regeneration with deciduous tooth stem cells, dental pulp stem cells, or bone-marrowderived mesenchymal stem cells for clinical study using tissue engineering technology. Tissue Eng Part A 2010;16:1891-1900.

10 Ma XW, Cui DP, Zhao DW: Vascular endothelial growth factor/bone morphogenetic protein-2 bone marrow combined modification of the mesenchymal stem cells to repair the avascular necrosis of the femoral head. Int J Clin Exp Med 2015;8:15528-15534.

11 Arbeloa-Gutierrez L, Dean CS, Chahla J, Pascual-Garrido C: Core Decompression Augmented With Autologous Bone Marrow Aspiration Concentrate for Early Avascular Necrosis of the Femoral Head. Arthrosc Tech 2016;5:e615-20.

12 Cao HJ, Guan HF, Lai YX, Qin L, Wang XL: Review of various treatment options and potential therapies for osteonecrosis of the femoral head. J Orthopaedic Translation 2016;4:57-70.

13 Wen Q, Ma L, Chen YP, Yang L, Luo W, Wang XN: Treatment of avascular necrosis of the femoral head by hepatocyte growth factor-transgenic bone marrow stromal stem cells. Gene Ther 2008;15:1523-35.

$>14$ Peng WX, Wang L, Deng J, Gong YK, Li SH, Hu YY: Application of BCBB/BMP/bFGF Complex in Repairing Femoral Head Necrosis in Rabbit Models. Journal of Hard Tissue Biology 2015;24:85-90.

-15 Tang TT, Lu B, Yue B, Xie XH, Xie YZ, Dai KR, Lu JX, Lou JR: Treatment of osteonecrosis of the femoral head with hBMP-2-gene-modified tissueengineered bone in goats. J Bone Joint Surg Br 2007;89:127-129.

-16 Zhang C, Ma J, Li M, Li XH, Dang XQ, Wang KZ: Repair effect of coexpression of the hVEGF and hBMP genes via an adeno-associated virus vector in a rabbit model of early steroid-induced avascular necrosis of the femoral head. Transl Res 2015;166:269-280.

-17 Katiella KA, Yanru Z, Hui Z: Magnesium alloy transfected BMSCs-BMP-2 composite in repair of femoral head necrosis with assessment of visceral organs. Springerplus 2016;5:1857.

18 Shi L, Sun W, Gao F, Cheng L, Li Z: Heterotopic ossification related to the use of recombinant human BMP2 in osteonecrosis of femoral head. Medicine (Baltimore) 2017;96:e7413.

19 Zhang HX, Zhang XP, Xiao GY: In vitro and in vivo evaluation of calcium phosphate composite scaffolds containing BMP-VEGF loaded PLGA microspheres for the treatment of avascular necrosis of the femoral head. Mater Sci Eng C Mater Biol Appl 2016;60:298-307.

20 Li B, Jha RK, Qi YJ, Ni QB, Wang H, Chen B, Chen LB: Early cellular responses of bmscs genetically modified with bfgf/bmp2 co-cultured with ligament fibroblasts in a three-dimensional model in vitro. Int J Mol Med 2016;38:1578-1586.

21 D'Mello S, Elangovan S, Salem AK: FGF2 gene activated matrices promote proliferation of bone marrow stromal cells. Arch Oral Biol 2015;60:1742-1749.

22 Chen B, Li B, Qi YJ, Ni QB, Pan ZQ, Wang H, Chen LB: Enhancement of tendon-to-bone healing after anterior cruciate ligament reconstruction using bone marrow-derived mesenchymal stem cells genetically modified with bFGF/BMP2. Sci Rep 2016;6:25940. 